\title{
Cefalópodos recolectados en la pesquería de crustáceos de la zona norte y centro-sur de Chile
}

\author{
Cephalopods collected in the Crustacean Fisheries from Northern and Central-Southern Chile \\ Juan C. Villarroel ${ }^{1}$, Marco A. Vega ${ }^{2 *}$ y Enzo Acuña ${ }^{1}$ \\ ${ }^{1}$ Departamento de Biología Marina, Facultad de Ciencias del Mar, Universidad Católica del Norte, Casilla 117, Coquimbo, Chile \\ jcvillar@nevados.ucn.cl \\ ${ }^{2}$ Departamento de Ciencias Ecológicas, Facultad de Ciencias, Universidad de Chile, Casilla 653, Santiago, Chile \\ *Dirección actual: Ecologia y Biodiversidad Marina, Instituto de Investigaciones Marinas, \\ Consejo Superior de Investigaciones Cientificas, \\ C/ Eduardo Cabello, 636208 Vigo, España.
}

\begin{abstract}
Resumen.- Se analizó la fauna de cefalópodos recolectados en la pesquería de arrastre de crustáceos entre 1996 y 1999. El área de estudio se localizó en el talud continental de Chile, entre los $21^{\circ} 34^{\prime}$ S y los $38^{\circ} 30^{\prime}$ S. Se registró un total de cinco taxa, siendo los pulpos Opisthoteuthis sp. y Benthoctopus sp. las especies con la mayor importancia relativa, mientras que los calamares Todarodes filippovae, Gonatus antarcticus y Neorossia sp. fueron menos frecuentes en las capturas. La mayor biomasa relativa de Opisthoteuthis sp., Benthoctopus sp. y T. filippovae se detectó al norte de los $30^{\circ} \mathrm{S}$ y $G$. antarcticus tuvo su mayor foco de abundancia entre los $33^{\circ} \mathrm{y}$ $34^{\circ} \mathrm{S}$. Todas las especies fueron capturadas principalmente alrededor de los $400 \mathrm{~m}$ de profundidad en la zona norte del área de estudio, mostrando Benthoctopus sp. y G. antarcticus una disminución en la profundidad de captura hacia la zona centro y sur de Chile. Se discute la identidad de Benthoctopus sp. y la distribución de los cefalópodos encontrados.
\end{abstract}

Palabras claves: Cefalópodos, talud continental, distribución, pesquerías de crustáceos, fauna asociada.

\begin{abstract}
The fauna of cephalopods collected in the crustacean trawl fishery between 1996 and 1999 was analyzed. The study area includes the continental slope off Chile between $21^{\circ} 34^{\prime} \mathrm{S}$ and $38^{\circ} 30^{\prime} \mathrm{S}$. A total of five taxa were recorded, being the octopods Opisthoteuthis sp. y Benthoctopus sp. the species with a higher relative importance, while the squids Todarodes filippovae, Gonatus antarcticus and Neorossia sp. were caught less frequently. Higher relative biomass of Opisthoteuthis sp., Benthoctopus sp. and T. filippovae were detected northward of $30^{\circ} \mathrm{S}$ and $G$. antarcticus had its higher abundance ground between $33^{\circ}$ and $34^{\circ} \mathrm{S}$. All species were caught mainly around $400 \mathrm{~m}$ depth in the northern study area, showing Benthoctopus sp. and G. antarcticus a decrease in the capture depth towards Central and Southern Chile. The identity of Benthoctopus sp. and the distribution of the cephalopod species found are discussed.

Key words: Cephalopods, continental slope, distribution, crustacean fisheries, by-catch.
\end{abstract}

\section{Introducción}

Los cefalópodos constituyen una de las ocho clases del filum Mollusca, morfológicamente adaptados a condiciones de natación y predación activa. Habitan en todos los ambientes oceánicos, ocupando un papel importante en el ecosistema marino.

Actualmente existen alrededor de 650 especies de cefalópodos en el mundo, alrededor de 175 son consideradas recursos de interés comercial (Roper et al. 1984, Nesis 1987, Guerra 1992), debido principalmente a su abundancia, rápido crecimiento y a su valor nutritivo rico en proteínas y bajo en grasas (Guerra 1992, Martínez 1989, Roper et al. 1984).
La mayoría de estas especies son poco abundantes y han sido incompletamente estudiadas en cuanto a su taxonomía, distribución y pesquería (Roper et al. 1984, Nesis 1987, Rocha et al. 1991, Rocha 1997, Vega et al. 2000a y b).

En Chile se han registrado 92 especies (Rocha 1997, Valdovinos 1999), de las cuales tres son de interés pesquero: el calamar Loligo gahi Orbigny 1835, la jibia Dosidicus gigas (Orbigny 1835) y el pulpo Octopus mimus Gould 1852 (Sernapesca 1990-1999), mientras que 13 son consideradas recursos pesqueros potenciales no explotados y suelen capturarse como pesca incidental o "by-catch” en distintas pesquerías (Roper 1981). 
A lo largo de la plataforma y talud continental del norte y centro-sur de Chile se desarrolla una pesquería de arrastre sobre los crustáceos langostino amarillo Cervimunida johni Porter 1903, langostino colorado Pleuroncodes monodon (Milne-Edwards 1837) y camarón nailon Heterocarpus reedi Bahamonde 1955, en la cual aparecen esporádicamente cefalópodos como fauna acompañante (Acuña et al. 1997).

El desarrollo de las pesquerías de crustáceos en el último cuarto del siglo pasado permitió poner la atención sobre la distribución y ecología de invertebrados demersales del talud continental de Chile central usando como método de muestreo el arrastre de fondo (Yañez 1974, Yañez \& Barbieri 1974, Báez \& Andrade 1980, Andrade 1986, Andrade 1987). En este contexto se enmarca el presente trabajo, el cual es una contribución al conocimiento de la taxonomía, composición específica, distribución y abundancia de los algunos cefalópodos del talud continental de la zona norte y centro-sur de Chile.

\section{Material y métodos}

\section{Área y período de estudio}

El área de estudio se localiza entre los $21^{\circ} 34^{\prime} \mathrm{S}$ y los $38^{\circ} 30^{\prime} \mathrm{S}$ en la costa de Chile, abarcando alrededor de $1.890 \mathrm{~km}$ de largo. La plataforma continental relacionado con isobatas del sector alcanza generalmente entre 5 y $10 \mathrm{~km}$ de ancho, llegando a 30 $\mathrm{km}$ en pocos lugares al sur de Valparaíso (Fig. 1). La circulación geostrófica de esta parte del Pacífico sudoriental se caracteriza por la presencia de dos corrientes principales. Una corriente superficial que fluye de sur a norte, la corriente de Humboldt, la cual transporta agua subantártica de baja temperatura y salinidad. Entre ésta y sobre la plataforma continental y la parte superior del talud continental fluye una corriente subsuperficial en sentido opuesto, la corriente Chile-Perú o de Günther, que transporta agua de origen ecuatorial subsuperficial entre 100 y 400 m. Bajo ambas corrientes se encuentra el agua intermedia antártica entre los 400 y 1.000 m de profundidad, de origen subantártico y caracterizada por su baja temperatura (Brattström \& Johanssen 1983).

Tomando en cuenta la baja frecuencia de cefalópodos en los lances de pesca, se descartó el análisis temporal de los datos, mientras que los antecedentes de las capturas permitieron dividir el área de estudio de la siguiente manera:

Zona de Antofagasta: $21^{\circ} 34^{\prime} \mathrm{S}-2^{\circ}$ 59’ S; Zona de Caldera: $25^{\circ} 00^{\prime} \mathrm{S}-28^{\circ} 59^{\prime} \mathrm{S}$;

Zona de Coquimbo: $29^{\circ} 00^{\prime} \mathrm{S}-31^{\circ} 59^{\prime} \mathrm{S}$; Zona de Valparaíso: $32^{\circ} 00^{\prime} \mathrm{S}-34^{\circ} 59^{\prime} \mathrm{S}$; y

Zona de Talcahuano: $35^{\circ} 00^{\prime} \mathrm{S}-38^{\circ} 30^{\prime} \mathrm{S}$.
El período de estudio abarcó información obtenida de la operación de la flota arrastrera entre los años 1996 y 1999, en fondos aptos para la captura de camarón y langostinos.

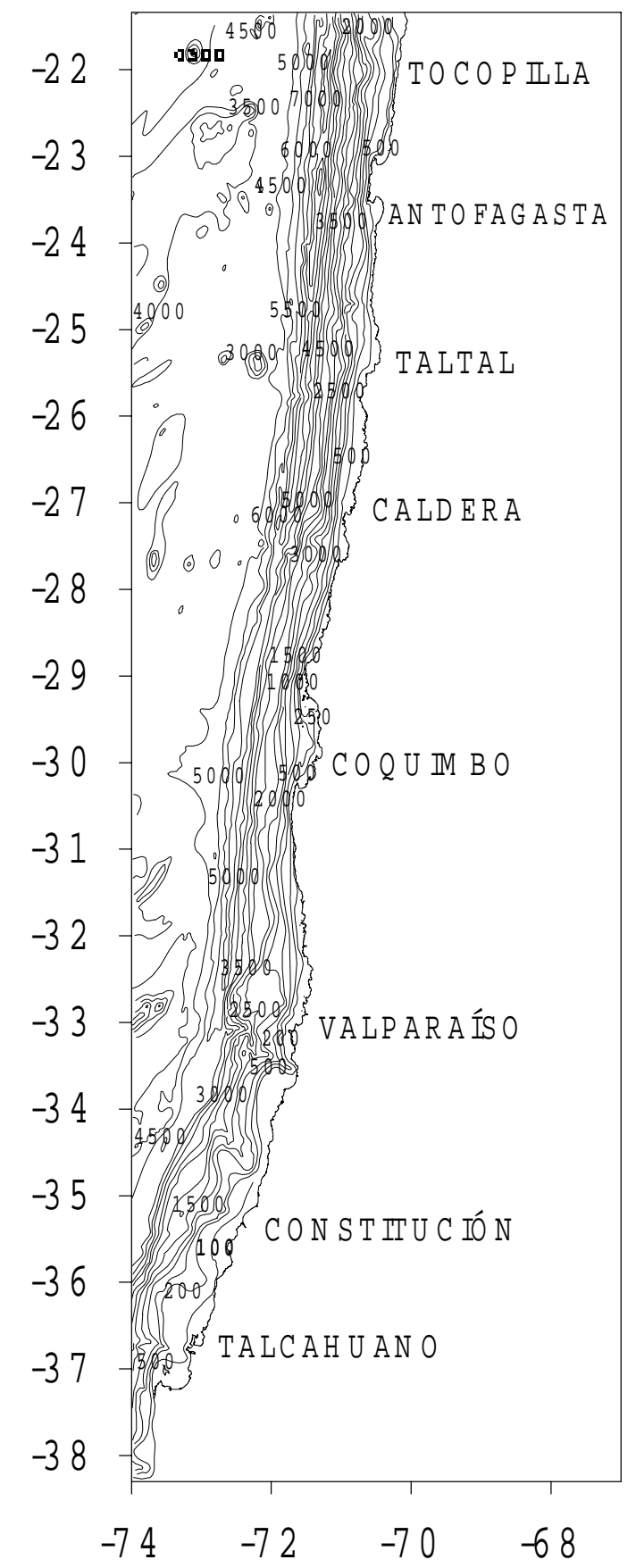

Figura 1

Área de estudio mostrando las isóbatas frente a la costa norte y centro-sur de Chile.

Study area showing isobats among to northern and centralsouthern coast off Chile. 


\section{Muestreo a bordo}

El método de muestreo de la fauna acompañante consistió en pesar los ejemplares con una pesa de precisión $1 \mathrm{~kg}$, cuando las condiciones lo permitieron. En caso contrario, o cuando la captura fue escasa se utilizó el método de cobertura (Acuña \& Arancibia 1996) que estima el porcentaje de una determinada especie de la fauna acompañante respecto al total de la captura obtenida en un lance de pesca. La captura total fue estimada por retrocálculo a partir de la fracción de la especie objetivo, cuyo peso se determina mediante el producto entre el peso promedio estándar de una caja apilable y el número total de cajas llenas. Las especies de cefalópodos recolectadas fueron mantenidas en hielo y luego llevados al laboratorio para su identificación.

\section{Identificación taxonómica}

En el laboratorio de Hidrobiología de la Facultad Ciencias de la Universidad de Chile los especímenes fueron medidos en su longitud dorsal del manto (LDM, $\mathrm{cm}$ ), fijados, preservados y puestos en frascos etiquetados y medios de conservación apropiados de acuerdo a las técnicas de fijación, conservación y curación para cefalópodos de Roper \& Sweeney (1983).

La identificación se realizó a través de los trabajos de Nesis (1987), Roper et al. (1984), Boletzky (1971) y Voss et al. (1998) mediante descripciones y diagnosis basadas en caracteres morfológicos de valor taxonómico específicos para cefalópodos (Roper \& Voss 1983). Un ejemplar de cada especie fue depositado en la colección de cefalópodos del Museo Nacional de Historia Natural (MNHN).

Las especies recolectadas fueron clasificadas (Anexo 1), confeccionándose posteriormente una clave taxonómica (Anexo 2) con apoyo de esquemas y términos para su uso (Anexo 3).

\section{Análisis de las capturas}

Se analizaron las bitácoras de pesca obtenidas en los embarques de personal científico, a bordo de embarcaciones arrastreras dedicadas a la extracción de crustáceos. El formulario de pesca contiene información relativa a las características del lance de pesca (posición geográfica y profundidad del lance, entre otros) y el peso de la captura de la especie objetivo y la fauna acompañante.

Con la información disponible se estimó la frecuencia de ocurrencia $(F O)$ del grupo de cefalópodos en los lances de pesca. Este registra el número de lances en que se encontró un taxón de cefalópodo, expresando dicho valor como un porcentaje respecto al número total de lances realizados por zona de pesca durante el período de estudio. También, se determinó la dominancia $(D)$ del taxón en la pesquería de la especie objetivo, que corresponde a la razón entre el número de lances con el taxón y el número total de lances en que se encontraron cefalópodos, expresada como porcentaje.

Los datos de la captura en peso fueron estandarizados mediante un índice de biomasa relativa para cada especie, la captura por unidad de esfuerzo (CPUE), la cual es una medida indirecta de la abundancia de los recursos pesqueros (Sparre \& Venema 1995). Debido a que la velocidad de arrastre se mantiene constante a una velocidad aproximada de dos nudos, entonces la CPUE se expresó en kilogramos por hora de arrastre $(\mathrm{kg} / \mathrm{h})$.

Análisis previos de los datos no evidenciaron que la distribución de la biomasa relativa se distribuyera lognormal, por lo tanto, se trabajó con la mediana que permitió determinar zonas de mayor biomasa relativa. De esta forma, el área de estudio fue subdividida cada un grado de latitud (unidad lineal) y sus medianas fueron estimadas. Los valores mayores a la mediana de todos los lances de pesca con un taxón determinado se consideraron como significativos, estableciendo zonas de mayor importancia. Cuando cada subdivisión no presentó suficientes valores entonces fue incorporada arbitrariamente a la subdivisión siguiente y posteriormente el resultado fue corregido, dividiendo dicho valor por el número de unidades lineales.

La distribución batimétrica de los lances con captura de cefalópodos se analizó con gráficos de cajas y bigotes. En estos gráficos la caja representa el 50\% de las observaciones, mientras que el bigote inferior y superior el 25\% restante, respectivamente. El punto dentro de la caja representa la mediana y los puntos más afuera de los bigotes son datos fuera de rango.

\section{Resultados}

Un total de cinco taxa de cefalópodos, correspondientes a tres especies y dos géneros se recolectaron en las pesquerías de arrastre de crustáceos, siendo los pulpos Opisthoteuthis sp. (Nesis 1987) y Benthoctopus sp. (Nesis 1987) las especies más relevantes en términos de importancia relativa (Tabla 1).

El 92,5\% de los cefalópodos se detectó en la pesca del camarón nailon, mientras que la fracción restante se obtuvo de las pesquerías de los langostinos amarillo y colorado. Ejemplares del género Benthoctopus fueron los más frecuentes y dominantes en las capturas de toda el área de estudio. Su mayor frecuencia de ocurrencia se encontró en las zonas de Caldera y Antofagasta, siendo la especie dominante al sur de Coquimbo (Tabla 2). La 
mayor biomasa relativa o CPUE de esta especie se encontró entre los $25^{\circ}$ y $28^{\circ} \mathrm{S}$, con valores superiores a $0,8 \mathrm{~kg} / \mathrm{h}$ y un máximo de $24 \mathrm{~kg} / \mathrm{h}$. Un foco de menor importancia también fue detectado entre los $29^{\circ}$ y $30^{\circ} \mathrm{S}$ con una biomasa relativa de mediana entre 0,4 y 0,5 kg/h y un máximo de 6,7 Kg/h (Fig. 2). Hacia el sur la CPUE disminuyó significativamente, encontrándose sólo lances de pesca aislados con altos valores.
El pulpo gelatinoso Opisthoteuthis sp. fue la especie con la más alta frecuencia y dominancia en la zona de Antofagasta y secundariamente en Caldera, desapareciendo al sur de Coquimbo (Tabla 2). Su mayor biomasa relativa se encontró entre los $25^{\circ}$ y $27^{\circ} \mathrm{S}$, zona de Caldera, con valores superiores a $1,8 \mathrm{~kg} / \mathrm{h}$ y un máximo de $27 \mathrm{~kg} / \mathrm{h}$. Un foco de menor importancia se detectó en los $22^{\circ} \mathrm{S}$, al norte de Antofagasta, con una mediana de $1,7 \mathrm{~kg} / \mathrm{h}$ y un máximo de 6,6 kg/h (Fig. 3).

Tabla 1

Captura anual (kg) e importancia relativa (\%) de los cefalópodos recolectados en la pesquería de crustáceos de la zona norte y centro sur de Chile.

Annual catch (kg) and relative importance (\%) of the cephalopods collected in the crustacean fisheries from northern and centralsouthern Chile.

\begin{tabular}{|c|c|c|c|c|c|c|c|c|c|c|}
\hline \multirow[b]{3}{*}{ especie } & \multicolumn{10}{|c|}{ año } \\
\hline & \multicolumn{2}{|c|}{1996} & \multicolumn{2}{|c|}{1997} & \multicolumn{2}{|c|}{1998} & \multicolumn{2}{|c|}{1999} & \multicolumn{2}{|c|}{ Total } \\
\hline & captura & $\%$ & captura & $\%$ & captura & $\%$ & captura & $\%$ & captura & $\%$ \\
\hline \multicolumn{11}{|l|}{ Opisthoteuthidae } \\
\hline Opisthoteuthis sp. & 419,1 & 36,5 & 7,5 & 14,0 & 41,9 & 30,7 & 456,8 & 70,0 & 925,3 & 46,4 \\
\hline \multicolumn{11}{|l|}{ Octopodidae } \\
\hline Benthoctopus sp. & 705,5 & 61,4 & 46,2 & 86,0 & 55,3 & 40,6 & 73,4 & 11,2 & 880,4 & 44,2 \\
\hline \multicolumn{11}{|l|}{ Ommastrephidae } \\
\hline Todarodes filippovae & 16,0 & 1,4 & & & 2,0 & 1,4 & 119,4 & 18,3 & 137,4 & 6,9 \\
\hline \multicolumn{11}{|l|}{ Gonatidae } \\
\hline Gonatus antarcticus & 8,5 & 0,7 & & & 37,2 & 27,3 & 3,1 & 0,5 & 48,8 & 2,5 \\
\hline \multicolumn{11}{|l|}{ Sepiolidae } \\
\hline Neorossia sp. & & & & & 0,1 & 0,1 & 0,3 & $<0,1$ & 0,1 & $<0,1$ \\
\hline Total & 1149,1 & 100 & 53,7 & 100 & 136,4 & 100 & 652,8 & 100 & 1992,0 & 100 \\
\hline
\end{tabular}

Tabla 2

Frecuencia de ocurrencia (FO) y dominancia (D) de los cefalópodos recolectadas en la pesquería de crustáceos de la zona norte y centro-sur de Chile.

Frequency of occurrence (FO) and dominance (D) of the cephalopods collected in the crustacean fisheries from northern and central-southern Chile

\begin{tabular}{|c|c|c|c|c|c|c|c|c|}
\hline \multirow[b]{3}{*}{ zona } & \multicolumn{8}{|c|}{ especies } \\
\hline & \multicolumn{2}{|c|}{ Benthoctopus sp. } & \multicolumn{2}{|c|}{ Opisthoteuthis sp. } & \multicolumn{2}{|c|}{ T. filippovae } & \multicolumn{2}{|c|}{ G. antarcticus } \\
\hline & $F O$ & $D$ & $F O$ & $D$ & $F O$ & $D$ & $F O$ & $D$ \\
\hline Antofagasta & 12,6 & 48,0 & 24,2 & 92,0 & 1,1 & 4,0 & 2,1 & 8,0 \\
\hline Caldera & 17,0 & 59,3 & 17,3 & 60,2 & 4,2 & 14,6 & 1,7 & 5,8 \\
\hline Coquimbo & 7,8 & 77,8 & 0,3 & 2,6 & 2,6 & 25,6 & 0,6 & 6,0 \\
\hline Valparaíso & 6,6 & 69,4 & & & 0,2 & 2,0 & 3,5 & 36,7 \\
\hline Talcahuano & 4,1 & 83,3 & & & & & 0,8 & 16,7 \\
\hline Total & 10,2 & 50,7 & 6,0 & 29,8 & 2,4 & 11,9 & 1,5 & 7,5 \\
\hline
\end{tabular}




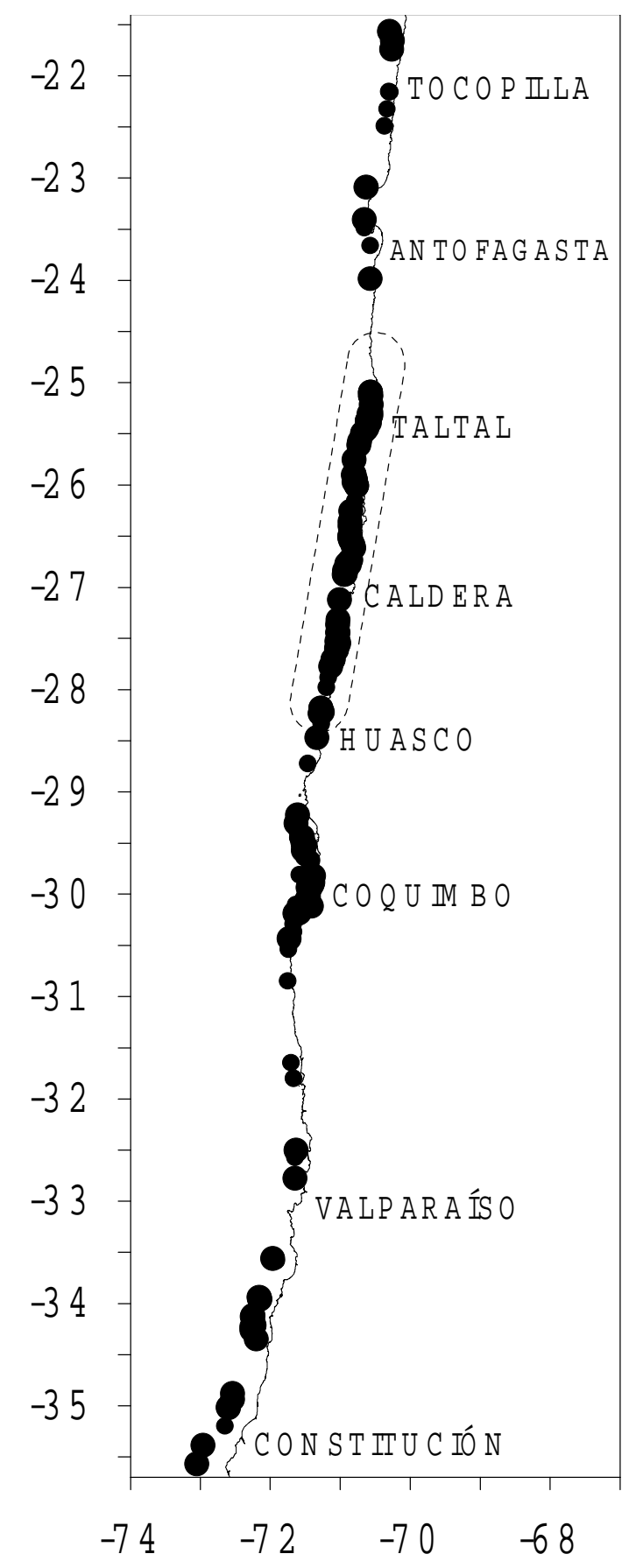

Figura 2

Biomasa relativa de Benthoctopus sp. obtenida como fauna acompañante desde la pesquería de crustáceos de la zona norte y centro-sur de Chile. Círculos grandes y pequeños representan una captura mayor y menor a la mediana $(0,6$ kg/h), respectivamente.

Relative biomass of Benthoctopus sp. obtained as by-catch of the crustacean fisheries from northern and central-southern Chile. Larger and smaller circles represent a higher and lower capture than the median value $(0.6 \mathrm{~kg} / \mathrm{h})$, respectively.

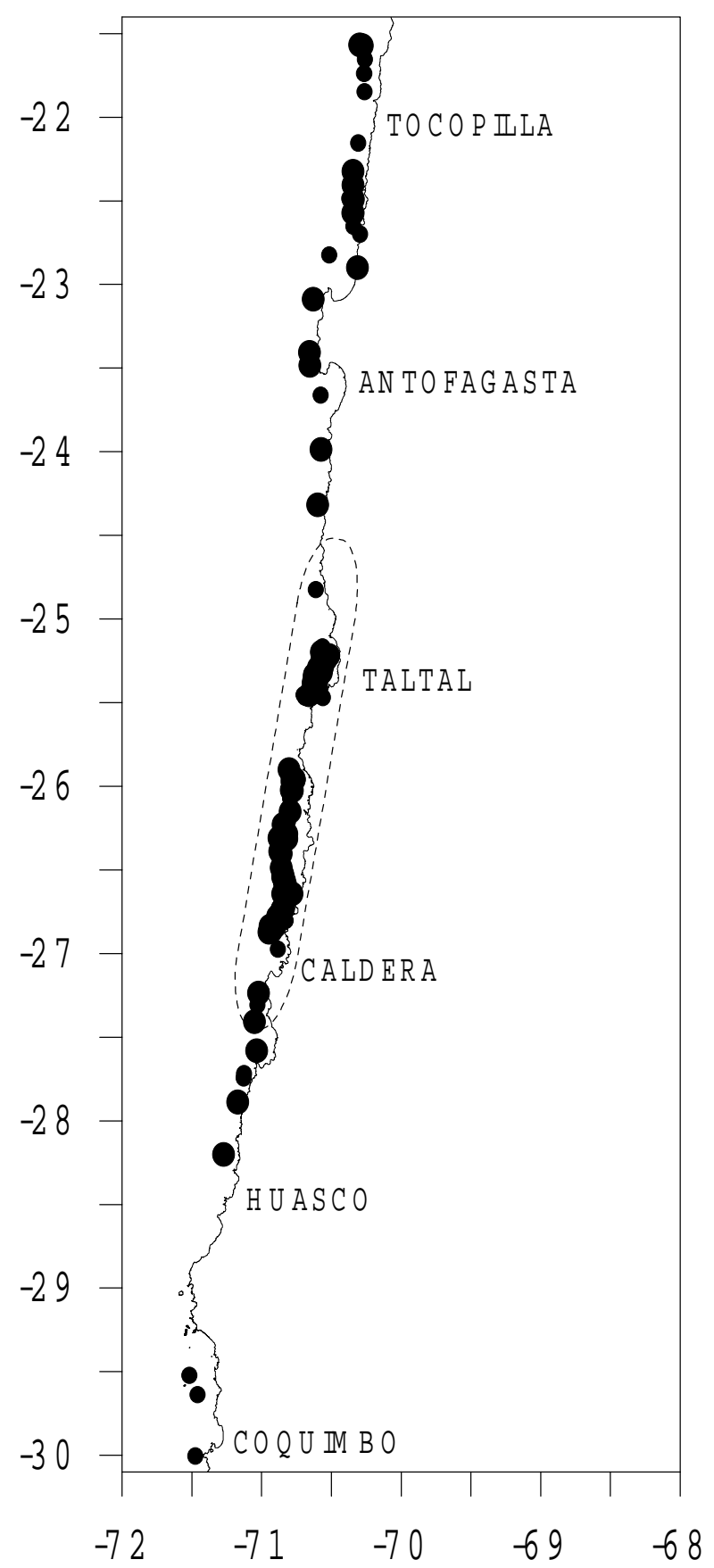

Figura 3

Biomasa relativa de Opisthoteuthis sp. obtenida como fauna acompañante desde la pesquería de crustáceos de la zona norte y centro-sur de Chile. Círculos grandes y pequeños representan una captura mayor y menor a la mediana $(1,8 \mathrm{~kg} / \mathrm{h})$, respectivamente.

Relative biomass of Opisthoteuthis sp. obtained as by-catch of the crustacean fisheries from northern and central-southern Chile. Larger and smaller circles represent a higher and lower capture than the median value $(1.8 \mathrm{~kg} / \mathrm{h})$, respectively. 


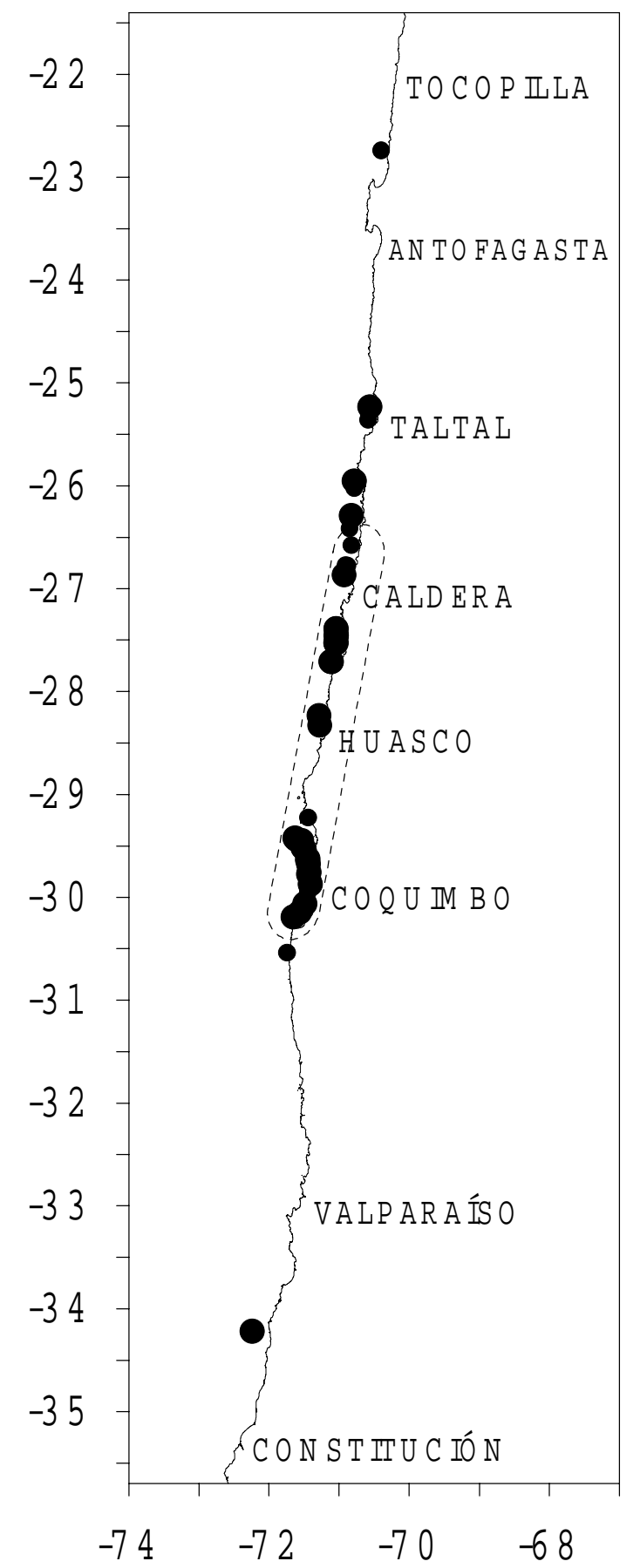

Figura 4

Biomasa relativa de Todarodes filippovae obtenida como fauna acompañante desde la pesquería de crustáceos de la zona norte y centro-sur de Chile. Círculos grandes y pequeños representan una captura mayor y menor a la mediana $(0,6 \mathrm{~kg} / \mathrm{h})$, respectivamente.

Relative biomass of Todarodes filippovae obtained as by-catch of the crustacean fisheries from northern and central-southern Chile. Larger and smaller circles represent a higher and lower capture than the median value $(0.6 \mathrm{~kg} / \mathrm{h})$, respectively.

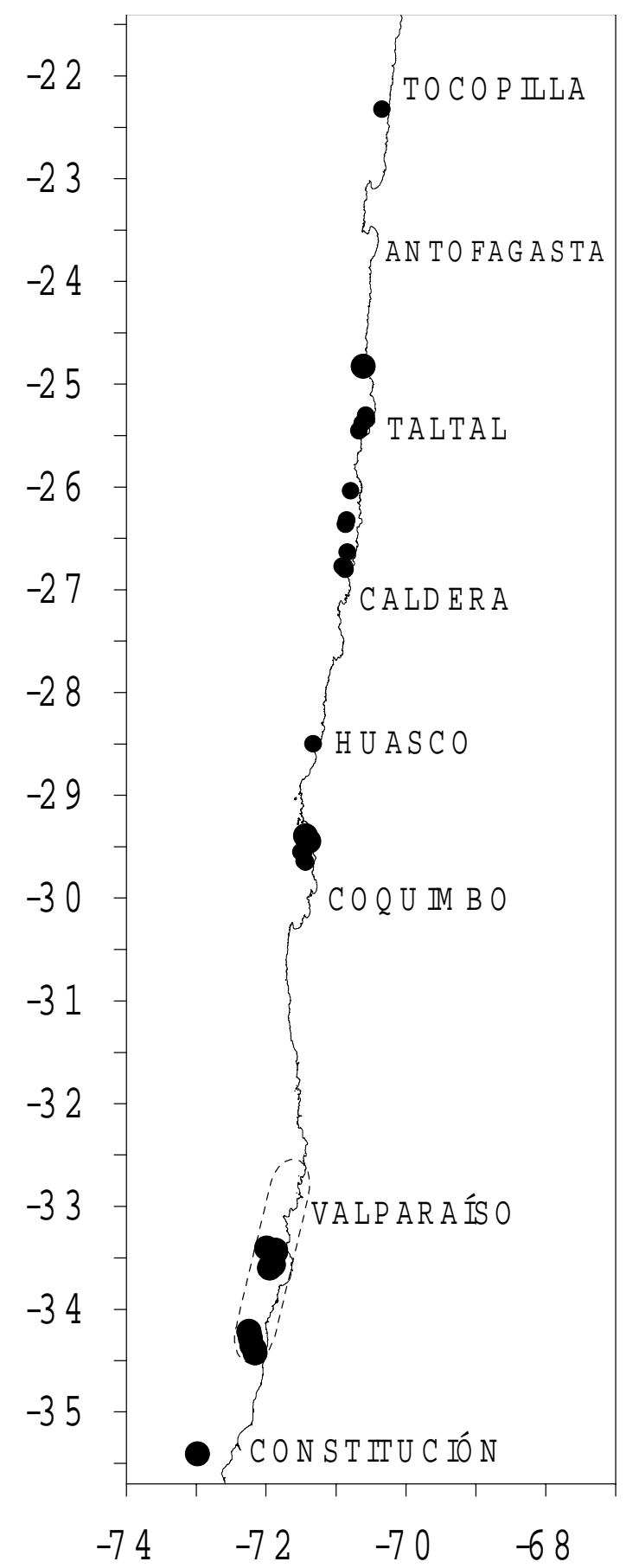

Figura 5

Biomasa relativa de Gonatus antarcticus obtenida como fauna acompañante desde la pesquería de crustáceos de la zona norte y centro-sur de Chile. Círculos grandes y pequeños representan una captura mayor y menor a la mediana $(0,3 \mathrm{~kg} / \mathrm{h})$, respectivamente.

Relative biomass of Gonatus antarcticus obtained as by-catch of the crustacean fisheries from northern and central-southern Chile. Larger and smaller circles represent a higher and lower capture than the median value $(0.3 \mathrm{~kg} / \mathrm{h})$, respectively. 
Los calamares Todarodes filippovae Adam 1975 y Gonatus antarcticus Lönnberg 1898 fueron menos frecuentes y dominantes que los pulpos mencionados más arriba. T. filippovae tuvo su mayor frecuencia de ocurrencia en Caldera, siendo la segunda especie dominante en la zona de Coquimbo. Mientras que $G$. antarcticus fue más frecuente y segunda en dominancia en la zona de Valparaíso (Tabla 2). T. filippovae presentó la mayor biomasa relativa entre los $27^{\circ}$ y $30^{\circ} \mathrm{S}$, con valores superiores a los $0,6 \mathrm{~kg} / \mathrm{h}$ y un rendimiento máximo de 3,4 kg/h (Fig. 4). En tanto, G. antarcticus tuvo su foco de mayor importancia entre $\operatorname{los} 33^{\circ}$ y $34^{\circ} \mathrm{S}$, zona de Valparaíso, con valores mayores a $0,3 \mathrm{~kg} / \mathrm{h}$ y un máximo de 5,5 kg/h (Fig. 5).

La distribución batimétrica de los lances de pesca realizados en la pesquería de crustáceos se muestra en la

(a)

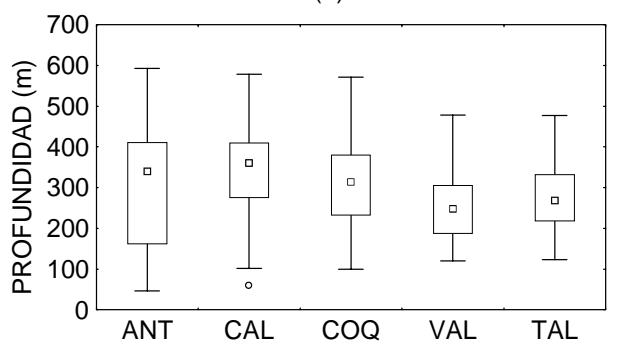

(c)

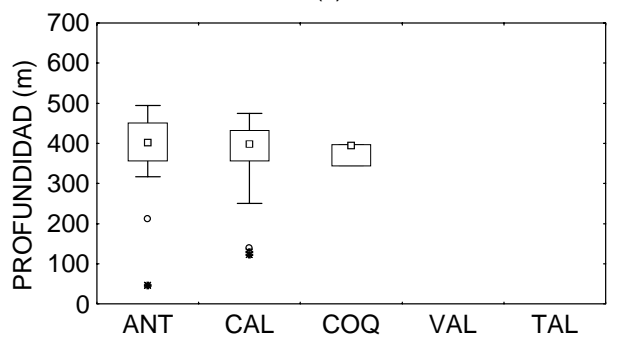

Figura 6. En general la mitad de los lances totales fueron realizados entre los 200 y $400 \mathrm{~m}$ de profundidad. La mediana en las zonas más al norte, Antofagasta y Caldera, se encuentra alrededor de los $350 \mathrm{~m}$ de profundidad, disminuyendo hacia el sur y tomando valores menores y cercanos a los $300 \mathrm{~m}$ de profundidad en Valparaíso y Talcahuano, respectivamente (Fig. 6a). Todas las especies de cefalópodos fueron capturadas alrededor de los $400 \mathrm{~m}$ de profundidad entre Antofagasta y Coquimbo (Fig. 6b-e). Además, Benthoctopus sp. y $G$. antarcticus mostraron una disminución en la profundidad de captura con la latitud, siendo en este último más pronunciada, encontrándose alrededor de los $200 \mathrm{~m}$ de profundidad en la zona de Valparaíso (Fig. 6e).

(b)

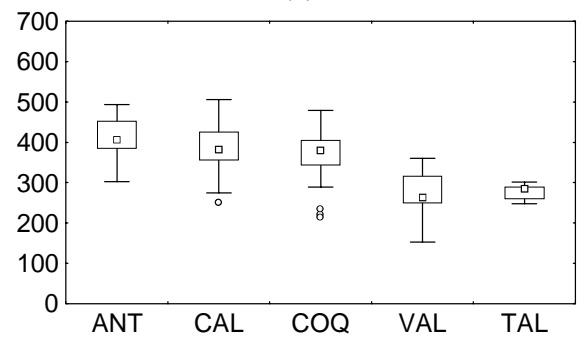

(d)

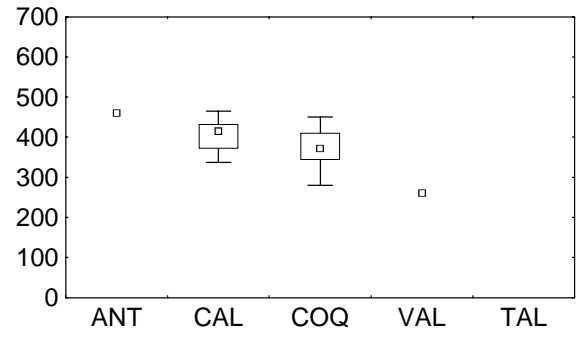

(e)

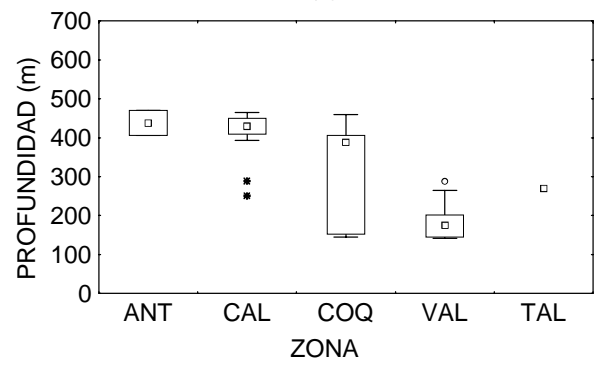

Figura 6

Distribución batimétrica de los lances (a) totales realizados en la pesquería de crustáceos de la zona norte y centro-sur de Chile; y con captura de (b) Benthoctopus sp., (c) Opisthoteuthis sp., (d) Todarodes filippovae y (e) Gonatus antarcticus, período 1996-1999. ANT, Antofagasta; CAL, Caldera; COQ, Coquimbo; VAL, Valparaíso; TAL, Talcahuano.

Bathymetric distribution of (a) total hauls trawled in the crustacean fisheries from northern and central-southern Chile; and with capture of (b) Benthoctopus sp., (c) Opisthoteuthis sp., (d) Todarodes filippovae y (e) Gonatus antarcticus, 1996-1999 period. ANT, Antofagasta; CAL, Caldera; COQ, Coquimbo; VAL, Valparaíso; TAL, Talcahuano. 
El calamar Neorossia sp. fue la especie con los más bajos valores de importancia relativa (Tabla 1), restringiéndose su captura a la zona de Caldera entre 339 y 407 m de profundidad.

El número de ejemplares de cefalópodos recolectados no fue suficiente para confeccionar distribuciones de frecuencia de talla. Sin embargo, se puede señalar que los especímenes de G. antarcticus no superaron los $8 \mathrm{~cm}$ LDM. El tamaño de Opisthoteuthis sp. fluctuó entre 29 y $45 \mathrm{~cm}$, con una media de $38 \mathrm{~cm}$ LDM. Benthoctopus sp. alcanzó tamaños de hasta $10 \mathrm{~cm}$ LDM y T. filippovae valores cercanos a los $35 \mathrm{~cm}$ LDM.

\section{Discusión}

La mayoría de las taxa de cefalópodos recolectadas en la pesquería de crustáceos de la zona norte y centro-sur de Chile, corresponden a especies comunes para aguas chilenas. Las cinco especies de cefalópodos obtenidas en este estudio corresponden a las más abundantes en la fauna acompañante, pudiendo haber quedado fuera otras de muy baja incidencia. Andrade $(1986,1987)$ señala un mayor número de especies en faenas de pesca realizadas anteriormente en la zona central de Chile, sin embargo actualmente se sabe que algunas de estas corresponden a otras especies. Octopus vulgaris Cuvier 1797 corresponde a Octopus mimus Gould 1852, mientras otras son especies dudosas en nuestras aguas tales como Rossia mastigophora Berry 1911 y Onychoteuthis borealijaponicus Okada 1927.

La presencia de Benthoctopus sp. ha sido señalada anteriormente en el norte de Chile (Cortéz 1992). Basándose en la morfología del hectocótilo tales como los índices de la longitud de la lígula y el cálamo, se sugiere al menos la presencia de dos nuevas especies para Chile del pulpo Benthoctopus sp. Ninguna de estas se parece a $B$. magellanicus citado para aguas del sur de Chile por Rocha (1997) y Valdovinos (1999). Tampoco a Benthoctopus sp. aff. januarii (Nesis 1973) citada por Nesis (1987) para la región este del Pacífico, en el norte de Perú.

El pulpo Opisthoteuthis sp. ha sido citado para el norte de Chile desde los $18^{\circ} 29^{\prime} \mathrm{S}$ hasta los $30^{\circ} \mathrm{S}$ (Rocha 1997, Valdovinos 1999). Nuestros resultados confirman la presencia de una importante biomasa al norte de Coquimbo, siendo el cefalópodo más frecuente en las capturas del camarón nailon.

El calamar Todarodes filippovae es una especie oceánica citada para aguas del centro y sur de Chile y circumpolar en el océano austral (Rocha 1997, Valdovinos 1999, Roper et al. 1984). Nuestros resultados muestran que esta especie también habita con una importante biomasa entre Caldera y Coquimbo.

El calamar Gonatus antarcticus es una especie que habita aguas oceánicas a lo largo de Chile desde los $42^{\circ}$ S hasta los 56 S (Rocha et al. 1991, Rocha 1997, Valdovinos 1999) y en aguas antárticas (Roper et al. 1988). En este estudio, se demostró su presencia hasta Tocopilla e incluso se observó una disminución de la biomasa hacia el norte con la mayor biomasa relativa entre los 33 y $34^{\circ} \mathrm{S}$.

Según la literatura, los pulpos Benthoctopus sp. y Opisthoteuthis sp. habitan principalmente aguas profundas de entre 600 y $2.000 \mathrm{~m}$ de profundidad (Voss 1967, Nesis 1987). Entre los 640 y los 720 m de profundidad se encuentra el núcleo de la masa de agua intermedia antártica caracterizada por su baja temperatura y salinidad y por un máximo relativo de oxígeno disuelto, cuyo límite superior se encuentra alrededor de los 400 m (Silva \& Sievers 1981, Brattström \& Johanssen 1983, Silva 1983). Este límite coincide con la mayor frecuencia de captura de ambas especies entre Caldera y Coquimbo. Sin embargo, no se descarta la presencia de una biomasa importante bajo esta profundidad, la cual no es normalmente utilizada por la pesquería de crustáceos.

Por otro lado, entre los 100 y los $200 \mathrm{~m}$ de profundidad se encuentra el núcleo de la masa de agua ecuatorial subsuperficial con su límite inferior alrededor de los 400 m (Silva \& Sievers 1981, Brattström \& Johanssen 1983, Silva 1983). Esta agua se caracteriza por un máximo de salinidad asociado a un mínimo de oxígeno disuelto, característica que puede explicar el hecho que estos especímenes no se distribuyan a menores profundidades.

Los calamares $G$. antarcticus y $T$. filippovae habitan desde la superficie hasta los 800 y $1.000 \mathrm{~m}$ de profundidad, respectivamente, siendo más abundantes en la capa superior a los 250 m (Roper 1981, Roper et al. 1984, 1988). La mayor frecuencia de lances con captura de $T$. filippovae, al igual que los otros cefalópodos, también se localizó alrededor de los 400 $\mathrm{m}$, no ajustándose estos resultados a lo señalado en la literatura. Sin embargo, es probable que en este caso el arte de pesca no sea el método de muestreo adecuado para explicar la distribución batimétrica de esta especie.

La distribución batimétrica de $G$. antarcticus coincide con lo señalado por otros autores, sólo en la zona de Valparaíso, donde se observó la mayor biomasa relativa de esta especie alrededor de los $200 \mathrm{~m}$ de profundidad.

La distribución batimétrica de Benthoctopus sp. está asociada a la presencia de aguas frías con un máximo relativo de oxígeno, coincidiendo con Silva \& Sievers 
(1981), quienes observaron una variación del núcleo de la masa de agua subsuperficial, el cual aumenta con la profundidad hacia el sur como consecuencia de su desplazamiento entre las masas de agua subantártica e intermedia antártica. Esto provoca su enfriamiento por erosión y mezcla. En consecuencia, el espesor de esta masa de agua es más grueso hacia el norte y el límite superior del agua intermedia antártica disminuye de profundidad hacia el sur.

Los ejemplares de Neorossia sp. representan la primera cita para aguas chilenas y es afín a Neorossia caroli (Joubin, 1902). Esta especie habita entre $300 \mathrm{y}$ 1.750 metros (Guerra 1992), principalmente en la zona batial intermedia (Nesis 1987), lo cual concuerda con la profundidad de los lances en que fue capturada.

De todas las especies recolectadas ninguna constituye un recurso de interés pesquero para Chile, aunque, se cree que $T$. filippovae si puede presentar algún potencial en este sentido (Roper et al. 1988). En aguas extranjeras, esta especie se captura con poteras y redes de arrastre, siendo de excelente calidad alimenticia (Roper 1981).

En general, la longitud dorsal del manto (LDM) de los ejemplares de $G$. antarcticus fue menor a la longitud máxima indicada para esta especie de $35 \mathrm{~cm}$ (Roper et al. 1988). Mientras que T. filippovae alcanzó valores cercanos a su máxima LDM de $50 \mathrm{~cm}$, más comúnmente entre 20 y $40 \mathrm{~cm}$ (Roper et al. 1984). En tanto las longitudes dorsales de manto de Opisthoteuthis sp. y Benthoctopus sp. muestran tallas habituales en nuestras aguas.

Será necesario realizar nuevos estudios sobre los cefalópodos de estas zonas para poder aclarar la identidad de las especies de Benthoctopus y Opisthoteuthis, complementar la distribución de las taxa que habitan la plataforma y el talud continental y determinar el tipo de movimiento que realizan en la columna de agua, sin dejar de considerar la posibilidad que algunas pudieran constituir recursos pesqueros potenciales.

\section{Agradecimientos}

Queremos expresar nuestro agradecimiento al Sr. Ricardo Catalán por su esmerado esfuerzo en la recolección de ejemplares a bordo, a los Srs. Sergio Letelier y Esteban Carreño del Museo Nacional de Historia Natural y a todas aquellas personas que en algún momento colaboraron. Esta investigación fue parcialmente financiada por los proyectos del Fondo de Investigación Pesquera FIP 95-06, 96-08, 97-25, 98-06 y 99-06; y por el programa de investigación "Monitoreo de la pesquería de crustáceos realizada por la flota de la
IV región” financiado por la Asociación de Industriales y Armadores Pesqueros IV Región A.G.

\section{Literatura citada}

Acuña E \& H Arancibia. 1996. Evaluación directa del stock del camarón nailon (Heterocarpus reedi) mediante el método del área barrida en la zona de operación de la flota arrastrera de las regiones III y IV. Informe Final Proyecto de Investigación. Depto. Biología Marina, U. C. del Norte Sede Coquimbo - Depto. Oceanografía, U. de Concepción, $71 \mathrm{p}$.

Acuña E, H Arancibia, R Roa, R Alarcón, C Díaz, A Mujica, F Winkler, I Lépez \& L Cid. 1997. Análisis de la pesquería y evaluación indirecta del stock de camarón nailon (II a VIII regiones). Informes Técnicos FIP, FIP-IT/ 95-06, $211 \mathrm{p}$.

Andrade H. 1986. Observaciones bioecológicas sobre invertebrados demersales de la zona central de Chile. En: P Arana (ed), La Pesca en Chile, p. 41-56. Universidad Católica de Valparaíso. Editorial Universitaria, Santiago, Chile.

Andrade H. 1987. Distribución batimétrica y geográfica de macroinvertebrados del talud continental de Chile central. Ciencia y Tecnología del Mar, CONA 11: 61-94.

Báez P \& H Andrade. 1980. Crustáceos decápodos asociados a la pesquería de Heterocarpus reedi Bahamonde 1955 en la zona central de Chile. Boletín del Museo Nacional de Historia Natural, Chile 37: 261-267.

Brattström H \& A Johanssen. 1983. Ecological and regional zoogeography of the marine benthic fauna of Chile. Report No. 49 of the Lund University Chile expedition 1948-49. Sarsia 68: 289-339.

Boletzky SV. 1971. Neorossia n.g. pro Rossia (Allorossia) caroli Joubin 1902 with remarks on the generic status of Semirossia Steenstrup 1887 (Mollusca; Cephalopoda). Bulletin of Marine Science 21: 964-969.

Cortéz T. 1992. Estudio taxonómico de los cefalópodos del norte de Chile. Informe Técnico $\mathrm{N}^{\circ} 1$. Proyecto UNAPSERPLAC, $71 \mathrm{p}$.

Guerra A. 1992. Mollusca: Cephalopoda. En: Ramos MA, J Alba, X Bellés, J Gonsálbez, A Guerra, E Macpherson, F Martin, J Serrano \& J Templado (eds), Fauna Ibérica. Vol. I. Museo Nacional de Ciencias Naturales (CSIC) Madrid, $327 \mathrm{p}$.

Martínez C. 1989. Los Cefalópodos nadadores: ¿Alternativa de diversificación para la pesquería chilena?. Chile Pesquero 39: 47-49.

Nesis KN. 1987. Cephalopods of the world, 351 p. T.F.H. Publications Inc., New Jersey.

Rocha F. 1997. Cephalopods in chilean waters, a review. Malacological Review 30: 101-113.

Rocha F, O Poblete \& N Bahamonde. 1991. Cefalópodos en contenidos gástricos de Merluccius australis polylepis Ginsburg y Macruronus magellanicus Lönnberg. Investigación Pesquera (Chile) 36: 51-65. 
Roper CFE. 1981. Cephalopods of the Southern Ocean Region: Potential Resources and Bibliography. Biological Investigations of Marine Antarctic Systems and Stocks, BIOMASS 2: 99-105.

Roper CFE \& GL Voss. 1983. Guidelines for taxonomic descriptions of cephalopod species. Memoirs of National Museum Victoria 44: 49-63.

Roper CFE \& MJ Sweeney. 1983. Techniques for fixation, preservation and curation of cephalopods. Memoirs of the National Museum Victoria 44: 29-47.

Roper CFE, MJ Sweeney \& CE Nauen. 1984. Cephalopods of the world. An annotated and illustrated catalogue of species of interest to fisheries. FAO Fisheries Synopsis 125(3): 1-277.

Roper C, M Sweeney \& M Clarke. 1988. Cefalópodos. En: Fischer W \& JC Hureau (eds), Océano Austral, área de la convención CCAMLR, áreas de pesca 48, 58 y 88, p. 117205. Fichas FAO de identificación de especies para los fines de pesca, FAO, Roma.

Silva N. 1983. Masas de agua y circulación en la región norte de Chile Latitudes $18^{\circ} \mathrm{S}-32^{\circ} \mathrm{S}$ (Operación Oceanográfica Marchile XI-Erfen II). Ciencia y Tecnología del Mar, CONA 7: 47-84.

Silva N \& H Sievers. 1981. Masas de agua y circulación en la rama costera de la Corriente de Humboldt Latitudes $18^{\circ} \mathrm{S}-$ $33^{\circ} \mathrm{S}$ (Operación Oceanográfica Marchile X-Erfen I). Ciencia y Tecnología del Mar, CONA 5: 5-50.

Sernapesca. 1990-1999. Anuario Estadístico de Pesca. Ministerio de Economía, Fomento y Reconstrucción.

Sparre P \& SC Venema. 1995. Introducción a la evaluación de recursos pesqueros tropicales. Parte 1. Manual. FAO Documento Técnico de Pesca. $\mathrm{N}^{\circ}$ 306.1, Rev.1. Roma. $440 \mathrm{p}$.
Sweeney MJ \& CFE Roper. 1998. Classification, type localities, and type repositories of recent cephalopoda. En: Voss GL, M Vecchione, RB Toll \& MJ Sweeney. Systematics and biogeography of cephalopods. Smithsonian Contributions to Zoology 586: 561-599.

Valdovinos C. 1999. Biodiversidad de moluscos chilenos: base de datos taxonómica y distribucional. Gayana 63: 111-164.

Vega MA, C Osorio \& F Rocha. 2000a. Indice bibliográfico sobre biodiversidad acuática de Chile: Cephalopoda (Mollusca). Ciencia y Tecnología del Mar, CONA 23: 139-144.

Vega MA, F Rocha \& C Osorio. 2000b. Comparación de las variaciones espaciales y temporales de las paralarvas de cefalópodos al norte y al sur de la constricción de Meninea en el canal de Moraleda (43 Lat. Sur). Ciencia y Tecnología del Mar, CONA 23: 69-82.

Voss GL. 1967. The biology and bathymetric distribution of deep-sea cephalopods. Studies of Tropical Oceanography 5: 511-535.

Voss GL, M Vecchione, RB Toll \& MJ Sweeney. 1998. Systematics and biogeography of cephalopods. Smithsonian Contributions to Zoology 586: 293-372.

Yañez E. 1974. Distribución y abundancia relativa estacional de los recursos disponibles a un arte de arrastre camaronero frente a la costa de Valparaíso (invierno y primavera 1972). Investigaciones Marinas, Valparaíso 5: 125-138.

Yañez E \& MA Barbieri. 1974. Distribución y abundancia relativa de los recursos disponibles a un arte de arrastre camaronero frente a la costa de Valparaíso (invierno 1973). Investigaciones Marinas, Valparaíso 5: 137-156. 


\section{Anexo 1}

Clasificación de los cefalópodos recolectados en este estudio (según Sweeney \& Roper 1998).

Clase CEPHALOPODA Cuvier 1797

Subclase COLEOIDEA Bather 1888

Superorden DECABRACHIA Boettger 1952

Orden SEPIOLIDA Fioroni 1981

Familia SEPIOLIDAE Leach 1817

Subfamilia ROSSIINAE Appellöf 1898

Género Neorossia Boletzky 1971

Neorossia aff. caroli (Joubin 1902)

Orden TEUTHIDA Naef 1916

Suborden OEGOPSINA Orbigny 1845

Familia GONATIDAE Hoyle 1886

Género Gonatus Gray 1849

Gonatus antarcticus Lönnberg 1898

Familia OMMASTREPHIDAE Steenstrup 1857

Subfamilia TODARODINAE Adam 1960

Género Todarodes Steenstrup 1880

Todarodes filippovae Adam 1975

Superorden OCTOBRACHIA Fioroni 1981

Orden OCTOPODIDA Leach 1818

Suborden CIRRINA Grimpe 1916

Familia OPISTHOTEUTHIDAE Verrill 1896

Género Opisthoteuthis Verrill 1883

Opisthoteuthis sp. Nesis 1987

Suborden INCIRRINA Grimpe 1916

Familia OCTOPODIDAE Orbigny 1840

Subfamilia BATHYPOLYPODINAE Robson 1928

Género Benthoctopus Grimpe 1921

Benthoctopus sp. Nesis 1987 


\section{Anexo 2}

Clave de ordenes, familias y géneros de cefalópodos recolectados en este estudio.

1. Con 10 apéndices circumorales (8 brazos y 2 tentáculos ventrolaterales); con ventosas pedunculadas provistas de anillos quitinosos, a veces garfios

Con 8 apéndices circumorales; ventosas pedunculadas o sésiles, con o sin anillos quitinosos, sin garfios 13 Orden Octopoda

2. Tentáculos retráctiles en bolsas situadas en la cabeza entre los brazos III y IV 3 Orden Sepiolida Tentáculos no retráctiles en bolsas; concha interna patente en forma de pluma 6 Orden Teuthida

3. Concha quitinosa interna reducida; cartílago de cierre del sifón con el manto sencillo recto .......4 Familia Sepiolidae

4. Borde ventral del manto sin proyectarse hacia delante; sólo brazos tercero y cuarto unido por una ancha umbrela. Borde dorsal del manto no fusionado con la cabeza 5 Subfamilia Rosiinae

5. Sin bolsa de la tinta. Papilas anales rudimentarias Género Neorossia (N. aff. caroli)

6. Ojos cubiertos por una membrana transparente (córnea) Suborden Myopsina Ojos sin córnea y en contacto directo con el agua 7 Suborden Oegópsina

7. Organo de cierre del sifón con el manto simple con una acanaladura y un resalte rectos. .8 Organos de cierre del sifón con el manto más complejo, no formando una acanaladura y un resalte rectos..... 10

8. Brazos con ventosas y/o garfios en 4 filas longitudinales 9 Familia Gonatidae

9. Con tentáculos bien desarrollados. Mano de la maza tentacular con un garfio grande al menos

Género Gonatus (G. antarcticus)

10. Cartílago de cierre en el sifón con hendidura transversal y otra longitudinal, formando una T invertida

11 Familia Ommastrephidae

11. Con faveola en la impresión sifonal pero sin bolsas laterales 12 Subfamilia Todarodinae

12. Membranas protectoras en los brazos desarrolladas normalmente, con trabéculas no protuberantes

Género Todarodes ( $T$. filippovae)

13. Con cirros en los brazos 14 Suborden Cirrata Sin cirros en los brazos 16 Suborden Incirrata

14. La cabeza no destaca del cuerpo, que está aplastado; aletas pequeñas; concha interna vestigial recta o curvada ligeramente 15 Familia Opisthoteuthidae

15. Brazos unidos por una umbrela que abarca $2 / 3$ de su longitud Género Opisthoteuthis

16. Hembras y machos de tamaños parecidos; hectocotilo no encerrado en bolsa y su extremo no filamentoso, sino formando una especie de cuchara (lígula). Las hembras carecen de expansiones en los brazos I y de cápsula incubadora 17 Familia Octopodidae

17. Sin bolsa de la tinta. Manto, cabeza y brazos sin tubérculos 18 Subfamilia Bathypolypodinae

18. Piel lisa; sin papilas supraoculares; lígula corta (5 a 7\% de la longitud del hectocotilo) Género Benthoctopus 


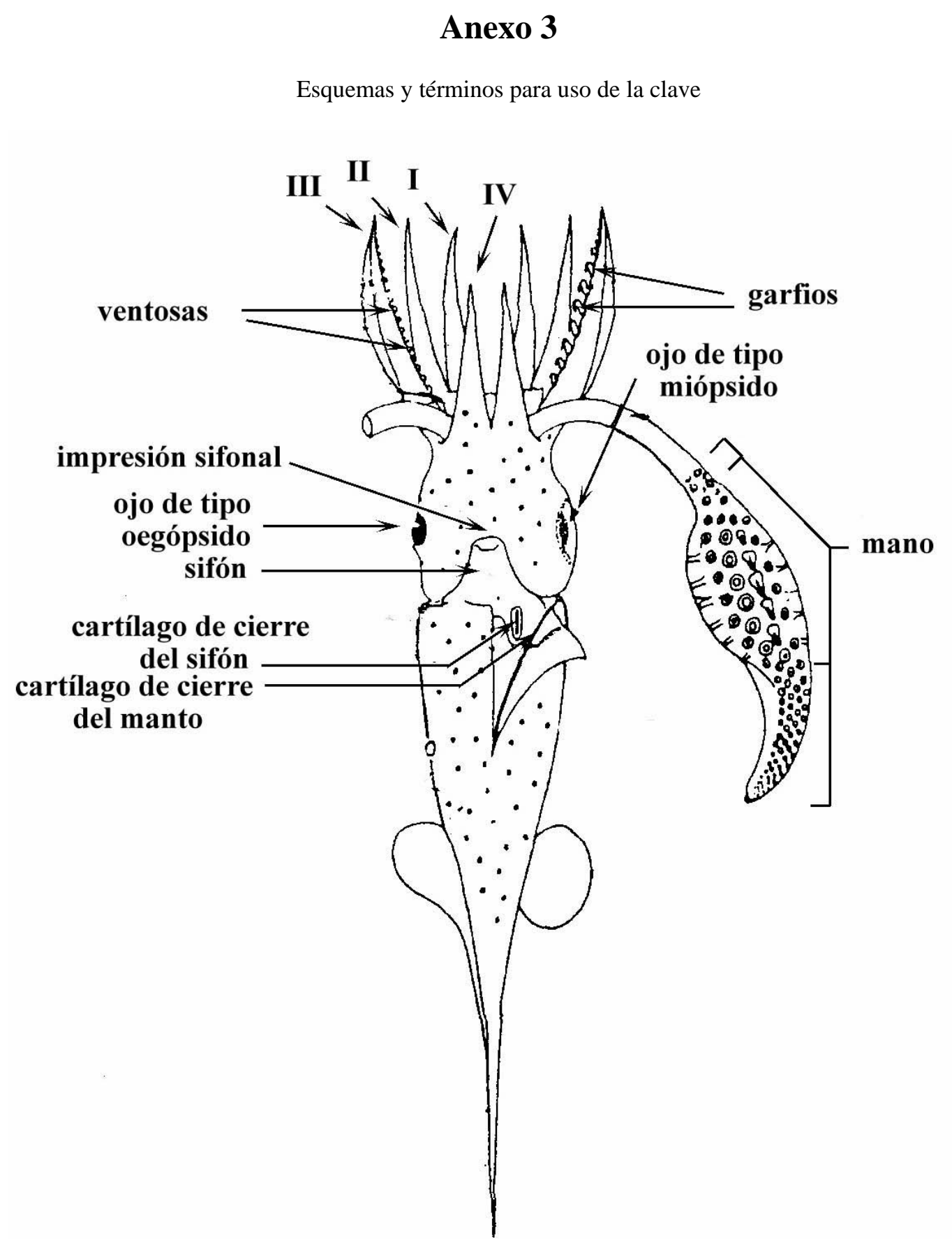

Esquema que ilustra las características más importantes de un calamar (teutoídeo) en vista ventral (modificado de Roper et al. 1988) 


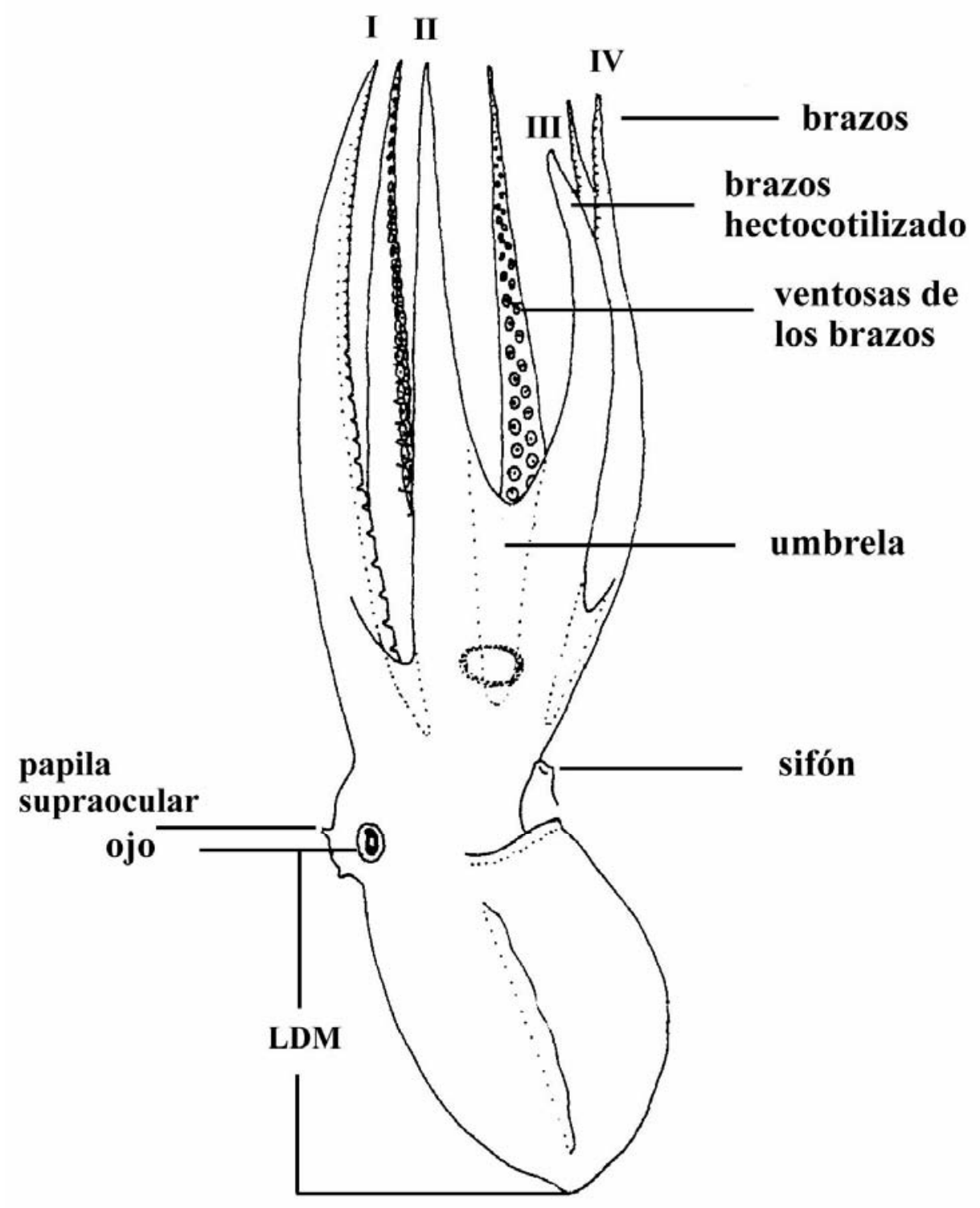

Esquema que ilustra los términos y medidas de un octópodo incirrado en vista lateral. LDM: longitud dorsal del manto (modificado de Guerra 1992). 


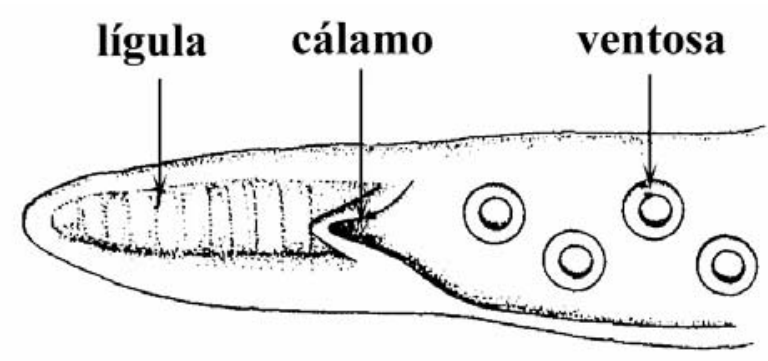

Región distal del hectocotilo de un octópodo incirrado (modificado de Roper et al. 1988)

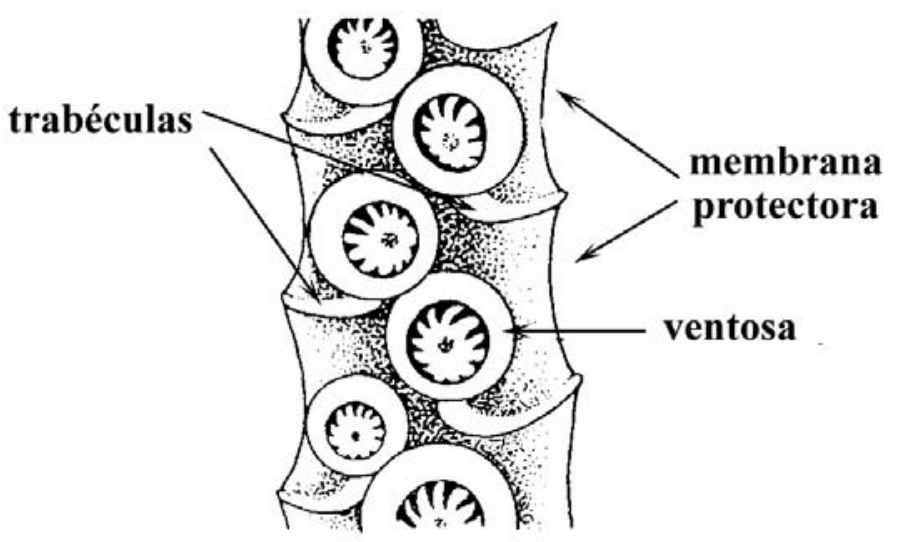

Estructuras presentes en los brazos o mazas tentaculares (modificado de Roper et al. 1988)

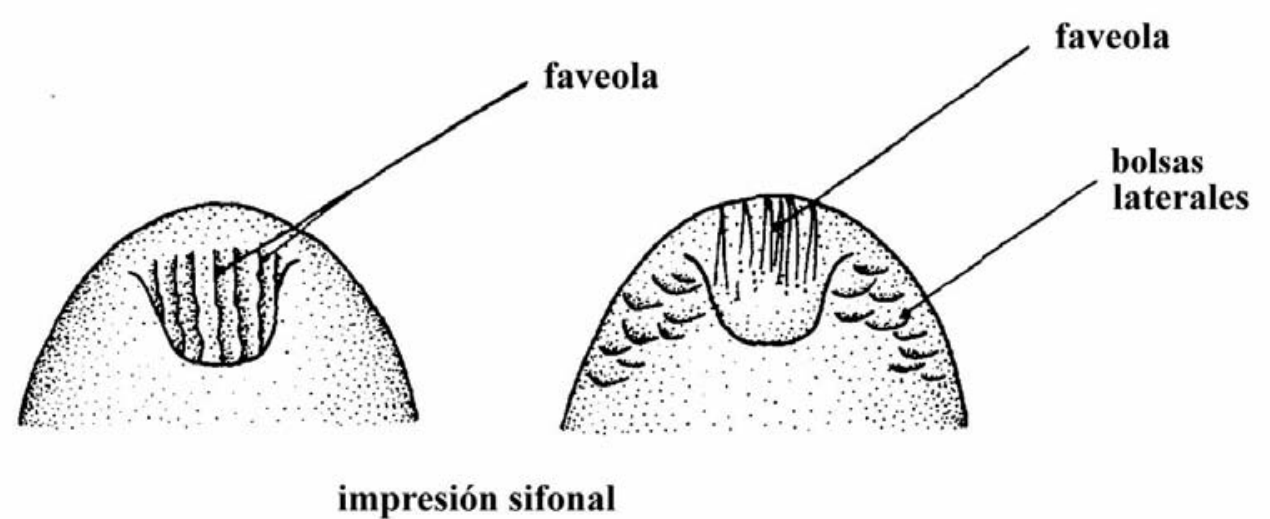

Estructuras presentes en la impresión sifonal de algunos oegópsidos (modificado de Guerra 1992) 